\title{
Experimental and theoretical studies of excited states in $\mathbf{I r}^{-}$
}

\author{
M. K. Kristiansson $\odot,{ }^{1, *}$ S. Schiffmann $\odot,{ }^{2,3}$ J. Grumer $\odot,{ }^{4}$ J. Karls $\odot,{ }^{5}$ N. de Ruette,,${ }^{1,6}$ G. Eklund, ${ }^{1}$ V. Ideböhn, ${ }^{5}$ \\ N. D. Gibson $\odot,{ }^{7}$ T. Brage $\odot,{ }^{2}$ H. Zettergren, ${ }^{1}$ D. Hanstorp,${ }^{5}$ and H. T. Schmidt ${ }^{1}$ \\ ${ }^{1}$ Department of Physics, Stockholm University, AlbaNova, SE-106 91 Stockholm, Sweden \\ ${ }^{2}$ Department of Physics, Division of Mathematical Physics, Lund University, Box 118, SE-221 00 Lund, Sweden \\ ${ }^{3}$ Spectroscopy, Quantum Chemistry and Atmospheric Remote Sensing (SQUARES), CP160/09, \\ Université Libre de Bruxelles (ULB), B 1050 Bruxelles, Belgium \\ ${ }^{4}$ Department of Physics and Astronomy, Theoretical Astrophysics, Uppsala University, Box 516, SE-751 20 Uppsala, Sweden \\ ${ }^{5}$ Department of Physics, University of Gothenburg, SE-412 96 Gothenburg, Sweden \\ ${ }^{6}$ European Spallation Source ERIC (ESS), SE-221 00 Lund, Sweden \\ ${ }^{7}$ Department of Physics and Astronomy, Denison University, Granville, Ohio 43023, USA
}

(Received 13 February 2021; accepted 20 May 2021; published 10 June 2021)

\begin{abstract}
The properties of atomic negative ions are to a large extent determined by electron-electron correlation which makes them an ideal testing ground for atomic many-body physics. In this paper, we present a detailed experimental and theoretical study of excited states in the negative ion of iridium. The ions were stored at cryogenic temperatures using the double electrostatic ion ring experiment facility at Stockholm University. Laser photodetachment was used to monitor the relaxation of three bound excited states belonging to the [Xe] $4 f^{14} 5 d^{8} 6 s^{2}$ ionic ground configuration. Our measurements show that the first excited state has a lifetime much longer than the ion-beam storage time of $1230 \pm 100 \mathrm{~s}$. The binding energy of this state was measured to be $1.045 \pm 0.002 \mathrm{eV}$. The lifetimes of the second and third excited states were experimentally determined to be $133 \pm 10$ and $172 \pm 35 \mathrm{~ms}$, respectively. Multiconfiguration Dirac-Hartree-Fock calculations were performed in order to extract binding energies and lifetimes. These calculations predict the existence of the third excited bound state that was detected experimentally. The computed lifetimes for the three excited bound states agree well with the experimental results and allow for a clear identification of the detected levels.
\end{abstract}

DOI: 10.1103/PhysRevA.103.062806

\section{INTRODUCTION}

The pronounced impact of electron-electron correlation on negative ions makes them fundamentally interesting and an important testing ground for the continued development of theoretical models of atomic systems with an accurate description of electron interactions [1,2]. A negative ion is formed when a neutral atom is polarized by the approach of an external electron. This results in an induced dipole moment of the atom that could be sufficiently strong to bind the extra electron. The stability of the negative ion depends on the amount of electron correlation within the ionic system and to what extent the electronic structure shields the attractive force from the nucleus. The weak and short-range nature of the polarization potential that occurs in the formation of a negative ion usually leads to only a single or a few bound states. Bound excited states, if they exist, in negative ions

\footnotetext{
*moa.kristiansson@fysik.su.se
}

Published by the American Physical Society under the terms of the Creative Commons Attribution 4.0 International license. Further distribution of this work must maintain attribution to the author(s) and the published article's title, journal citation, and DOI. Funded by Bibsam. usually belong to the same electronic configuration as the ground state and often to the same $L S$ term. To this day there are only four elements for which excited states with opposite parity to that of the ground state have been shown to exist, namely, in $\mathrm{La}^{-}, \mathrm{Os}^{-}, \mathrm{Ce}^{-}$, and $\mathrm{Th}^{-}$[3-7]. All other known bound excited states in negative ions have the same parity as the ground state and cannot relax radiatively through electric dipole $(E 1)$ transitions. Instead, they can decay radiatively through much slower higher-order modes, such as magnetic dipole $(M 1)$ or electric quadrupole $(E 2)$ transitions. This typically results in lifetimes that are significantly longer than lifetimes characteristic of E1-dominated states in negative ions $[3,4]$. Longer lifetimes are generally more difficult to measure, especially since negative ions are fragile and tend to decay when colliding with residual gas present in a particle trap or along a beam line [8-10].

One of the first studies of the lifetimes of excited states of a negative ion involved the autodetaching states of $\mathrm{He}^{-}$ [11]. These measurements were performed by monitoring the ion-beam current along a time-of-flight beam line. Other early work on lifetimes of excited states in negative ions was performed using beam-foil spectroscopy (see, e.g., Refs. [12,13]). In such a setup, accelerated ions pass through a metal foil where ions and neutral atoms are formed in excited states. The decays of these states are then monitored along a beam line by observing the fluorescence from the excited 
states as a function of the distance from the foil. A more efficient method for lifetime studies is by using ion-storage rings; a technique first implemented at the ASTRID storage ring [14]. In this magnetic storage ring operating at room temperature, lifetimes of excited states of negative atomic ions in the microsecond range were measured [15]. In order to store heavier ions and avoid magnetic fields affecting the measurements, the electrostatic ion-storage ring ELISA was constructed [16]. Lifetimes are also studied in tabletop electrostatic ion-beam traps where ions are reflected back and forth between electrostatic mirrors for extended periods of time as in a storage ring [17]. A cryogenic electrostatic ion-beam trap was used to perform the so far most accurate lifetime measurements of the autodetaching states of $\mathrm{He}^{-}$ [18]. Many other electrostatic storage rings and traps have since then been designed and used to study negative ions. Some of the most recent cryogenic storage ring experiments include RICE in Japan [19], CSR in Germany [20] and double electrostatic ion ring experiment (DESIREE) in Sweden [21]. DESIREE was used to determine a lifetime of $503 \pm 54 \mathrm{~s}$ for the ${ }^{2} P_{1 / 2}$ excited state of $S^{-}$: the longest lifetime measured of an excited state in a negative ion so far [8]. This was made possible by means of the extreme vacuum conditions, which can only be achieved in cryogenic environments.

Iridium (Ir) with atomic number 77 belongs to the platinum group, and it is one of the least abundant elements in the crust of the earth. A famous application for Ir is in the alloy used for the historical international meter and kilogram standards. It has two naturally occurring isotopes ${ }^{191}$ Ir and ${ }^{193} \mathrm{Ir}$, which both are stable and with natural abundances of $37 \%$ and $63 \%$, respectively. The atomic ground state of Ir is, in a configuration- and $L S$-coupling pure picture, [Xe] $4 f^{14} 5 d^{7} 6 s^{24} F_{9 / 2}$, which includes an open $d$-valence subshell. The ground state of the negative ion, [Xe] $4 f^{14} 5 d^{8} 6 s^{2}{ }^{3} F_{4}$, has an extra $d$ electron compared to the neutral system. For both the neutral and the ionic ground states, their ${ }^{2 S+1} L_{J}$ level identifications are solely determined by the partially filled $5 d$ subshell since $6 s^{2}$ represents a closed subshell. In the case of photodetachment from the ground state of $\mathrm{Ir}^{-}$to the ground state of the neutral Ir atom, an electron from the $5 d$ subshell is detached with $l=|2 \pm 1|=1$ or 3, i.e., as a $p$ wave or $f$ wave. As is always the case close to the detachment threshold, the $f$ wave is suppressed by the centrifugal barrier, and the $p$ wave will dominate the photodetachment cross section. The behavior of the cross-section $\sigma$ close above the threshold is described by the Wigner law [22]: $\sigma \propto E^{l+1 / 2}$, where $l$ and $E$ are the orbital angular momentum quantum number and the energy of the outgoing electron, respectively. As for all non-s-wave outgoing electrons, this gives a slow onset at threshold. The negative Ir ion has previously been studied using laser photodetachment and photoelectron spectroscopy [23-27]. In Fig. 1, we show an energy-level diagram of $\mathrm{Ir}^{-}$ and its bound excited states. As will be discussed in Sec. IV, the $\mathrm{Ir}^{-}$excited states are predicted to show large $L S$-term mixing, which prevents identification of single $L S$ terms. These states do, however, still have a good quantum number in $J$, the total angular momentum quantum number. We, therefore, choose to identify the states using the labels $X_{J}$ for the ground state and $A_{J}, B_{J}$, and $C_{J}$ for the excited states. The electron affinity is known to a high level of precision [24-27], and

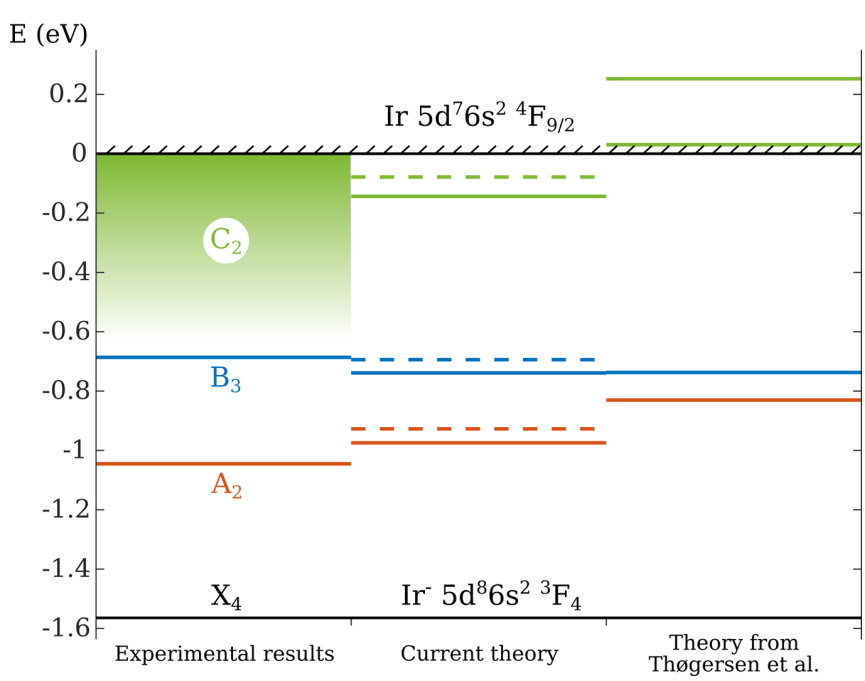

FIG. 1. Energy-level diagram for the bound states of $\mathrm{Ir}^{-}$. The vertical scale indicates energies relative to the ground state of the atom. $X_{4}$ is the ground state of the negative ion whereas $A_{2}, B_{3}$, and $C_{2}$ are the three bound excited states. Since no experimental determination of the binding energy of state $C_{2}$ exists, we represent it with a block gradient to indicate the experimentally possible range of its binding energy. The experimental results, shown in the left column, are from the current experiment as well as from several earlier experiments $[23,26,27]$. The experimental values for the binding energies are given and compared to the calculated energies in Table I. The theoretical calculations shown in the center column are from the current paper where the dashed lines correspond to energy levels from the valence-valence (VV) correlation model and the solid lines are those obtained using the expanded VV + core-valence (CV) model. Previous calculations labeled Theory from Thøgersen et al. [23] are shown in the right column [23].

the binding energy of the second excited state $B_{3}$ has been measured with high accuracy through two-photon detachment spectroscopy [23]. In the same study theoretical calculations predicted another bound state, labeled Theory from Thøgersen et al. [23] in Fig. 1. This first excited state $A_{2}$ was very recently observed, and its binding energy was measured via photoelectron spectroscopy [27]. The same study also performed an even more accurate determination of the electron affinity and a measurement of the binding energy of the second excited state $B_{3}$. There is a discrepancy in the measured binding energy of the $B_{3}$ state between the two-photon detachment spectroscopy [23] experiment and the photoelectron spectroscopy [27].

In the current paper we perform both measurements and theoretical calculations of the lifetimes of all possibly bound excited states of $\mathrm{Ir}^{-}$. The lifetimes are measured using the DESIREE facility by applying a beam of laser light to a beam of $\mathrm{Ir}^{-}$. Using this technique we perform time-resolved photodetachment spectroscopy on the stored ions. The calculations of binding energies and lifetimes are conducted using the variational fully relativistic multiconfiguration Dirac-Hartree-Fock (MCDHF) method [28] as implemented in the GRASP2018 package [29]. Heavy negative ions, such as $\mathrm{Ir}^{-}$are especially interesting systems from a theoretical point of view since both electron correlation and relativistic effects play an important role 




FIG. 2. Mass spectrum of the $\mathrm{Ir}^{-}$isotopes. The two naturally occurring isotopes of mass 191 and $193 \mathrm{u}$. There is also a small amount of beam current at mass 192 amu and 194 amu which we attribute to ${ }^{191} \mathrm{IrH}^{-}$and ${ }^{193} \mathrm{IrH}^{-}$, respectively.

[30,31]. Joint experimental and theoretical studies are essential to the understanding of how these effects influence binding energies and lifetimes (see, e.g., Refs. [32,33]).

\section{EXPERIMENTAL SETUP AND PROCEDURE}

In this paper, one of the two storage rings of DESIREE is used to study photodetachment of $\mathrm{Ir}^{-}$. We limit the presentation of the experimental setup to the specifics of the current measurement. General specifications and further information on the operation of DESIREE have been described in earlier publications $[8,21,34]$. To summarize, the circumference of each of the storage rings is $8.7 \mathrm{~m}$, including a common straight section. The entire system is kept at a temperature of $13.5 \mathrm{~K}$, which reduces the black-body radiation that could otherwise detach loosely bound states. Furthermore, it contributes to reduce the particle density of the rest gas in the vacuum chamber to a particle density of about $10^{4} \mathrm{~cm}^{-3}$. This allows for long storage times since an important loss mechanism of an anionic beam is residual gas collisions [21]. It is apparent from the current paper that improvements to the vacuum system of DESIREE now allows for storage times that are approaching a regime where the limiting factor for the storage lifetime is instrumental effects rather than residual-gas collisions. In this paper, a beam of $\mathrm{Ir}^{-}$is produced in a SNICS II cesium sputter source [35] and accelerated to $10 \mathrm{keV}$. The beam is mass selected using a $90^{\circ}$ bending magnet before being injected into one of the storage rings of DESIREE. A typical mass spectrum is shown in Fig. 2. The ${ }^{193} \mathrm{Ir}$ isotope is the most abundant one and is, therefore, selected for the experiment. Contributions from contaminants, such as ${ }^{191} \mathrm{IrH}_{2}^{-}$at mass 193 are found to be insignificant since the signal at mass 192 consisting of ${ }^{191} \mathrm{IrH}^{-}$is very small, and there is no visible signal at mass 195 corresponding to ${ }^{193} \mathrm{IrH}_{2}^{-}$. After operating the ion source for longer times, the signal at mass 192 disappears completely. Most of the measurements were repeated also for the ${ }^{191} \mathrm{Ir}$ isotope, which confirms with certainty that the signal comes from atomic Ir anions and not some contamination of the ion beam.
However, these measurements are of lower statistical quality and not used in the final analysis of the data. One exception is for the measurement of the photodetachment spectrum in Fig. 7 where the measurement is repeated for both isotopes, and the data shown are from ${ }^{191} \mathrm{Ir}$. The ion-beam current is recorded using a Faraday cup before injection into the storage ring. The beam current used in the experiment was in the range of 1 and $40 \mathrm{nA}$ depending on the type of measurement.

The photodetachment measurement is performed with two different laser configurations as illustrated in Fig. 3. Along the straight section where the ions are injected, a continuous-wave titanium sapphire laser beam is merged with the ion beam. The laser is applied in a counterpropagating direction with respect to the ions, and the neutral atoms formed in the photodetachment process are no longer confined within the storage ring and propagate straightforward until they hit the glass plate detector. This detector consists of an optically transparent conversion plate that produces secondary electrons when impacted by a neutral particle with kinetic energy in the $\mathrm{keV}$ range and a microchannel plate (MCP) used to detect secondary electrons. The conversion plate is a glass plate covered with thin layers of titanium and gold to optimize the electron yield and to prevent electric charge up. At the back of the $\mathrm{MCP}$, the timing and position information is processed by a resistive-anode encoder. The second photodetachment scheme involves a diode pumped nanosecond $Q$-switched $\mathrm{Nd}$ :yttrium aluminum garnet laser that operates at a $1-\mathrm{kHz}$ repetition rate. It is combined with an optical parametric oscillator (OPO) that allows for tuning the photon energy over a wide range from the UV to the near-IR region. The laser beam crosses the ion beam at a right angle at the center of the straight section of the ring (see Fig. 3), and the pulse energies of the photon energy intervals used are on the order of 50-100 $\mu \mathrm{J}$. Since the time of flight from the laser interaction region to the detector is known, the signal can be gated with respect to the collisional background allowing for even very weak signals to be identified [10]. The lifetimes and measured binding energy are obtained by using a standard nonlinear least-squares methodology and fitting the data to an exponential decay for the lifetimes or to the Wigner law in the case of the binding energy. The error bars given for the measured lifetimes and binding energy are one standard deviation.

\section{EXPERIMENTAL RESULTS}

In order to measure the storage lifetime of the ion beam, we use the technique of laser photodetachment of all bound states of the ion. The two excited states $A_{2}$ and $B_{3}$ have excitation energies larger than $0.5 \mathrm{eV}$ and in such cases only a minute fraction of the ions produced in a cesium sputtering source are excited, whereas most of the ions will be in the ground state. This is also clear in the signal obtained in our measurements in Fig. 4. If the number of ions in excited states with a lifetime much shorter than the storage time was high, one would see a clear deviation from a single exponential decay as the photodetachment cross sections are likely to be quite different for the different levels. This is, however, not the case since we see a clear single exponential decay and can, therefore, be confident that this is due to the signal being dominated by ions in the ground state that are being photodetached. An important 

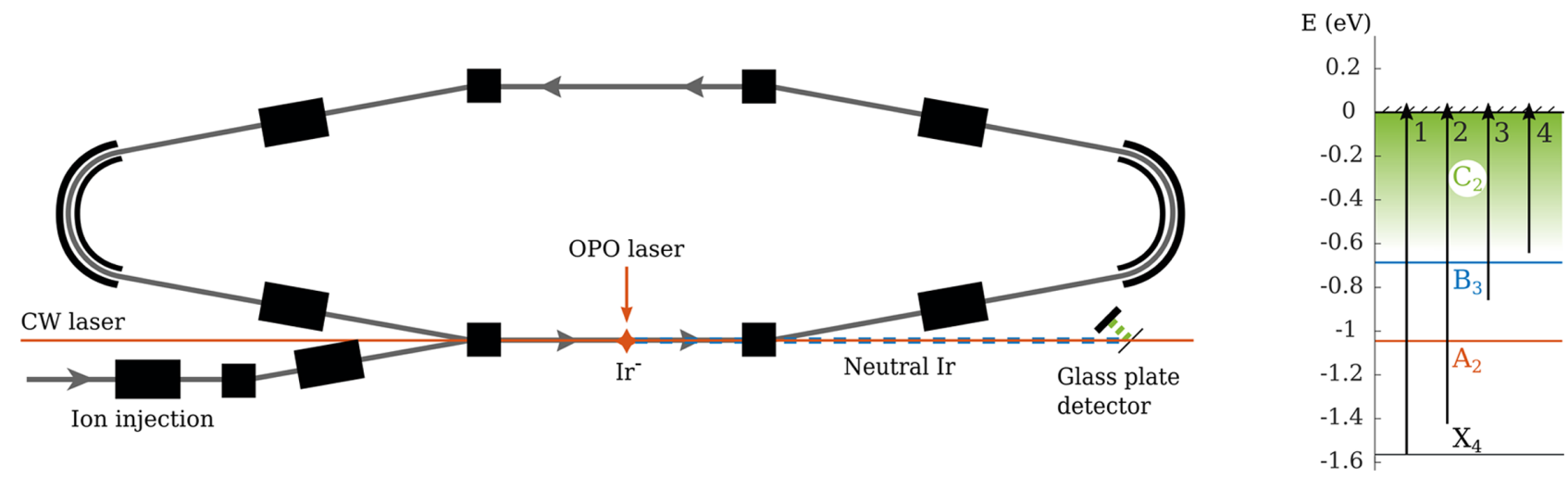

FIG. 3. Left: Schematic of one of the storage rings of DESIREE. $\mathrm{Ir}^{-}$ions of $10 \mathrm{keV}$ are injected into the ring and stored for up to $3000 \mathrm{~s}$. Photodetachment is performed either collinearly along the straight section of the ring or perpendicularly in the center of the same straight section. Ir atoms formed in the photodetachment process are detected as they continue to travel forward after the straight section. Right: The energy-level diagram of the excited states of $\mathrm{Ir}^{-}$. The vertical scale indicates energies relative to the ground state of the neutral atom. $X_{4}$ is the ground state of the negative ion, and $A_{2}, B_{3}$, and $C_{2}$ are the three excited states. The four arrows represent the four different photon energies used to probe different states in the experiment. Arrow number 1 corresponds to $1.591 \mathrm{eV}$, arrow number 2 corresponds to $1.459 \mathrm{eV}$, arrow number 3 corresponds to $0.8856 \mathrm{eV}$, and arrow number 4 corresponds to $0.6702 \mathrm{eV}$.

parameter in this type of experiment is the density of ions stored in the ring since a large ion beam density will shorten the storage lifetime due to ion-ion interactions [21]. In all measurements the ion-beam current was, therefore, reduced to a level where it no longer affected the measured lifetimes.

The measurement of the storage time is conducted using a photon energy of $1.591 \mathrm{eV}$, which corresponds to arrow no. 1 in the level diagram in the right part of Fig. 3. The titanium sapphire laser is used at a laser power set to result in a photodetachment signal strong enough for an efficient measurement yet weak enough to not significantly reduce the average storage lifetime of the ions. The laser light is chopped with a shutter that is open for $1 \mathrm{~s}$ to measure the photodetachment signal and closed for $9 \mathrm{~s}$ to measure the background. This method reduces the time that the ion beam is exposed to the laser light in order to minimize the effect of the laser on the

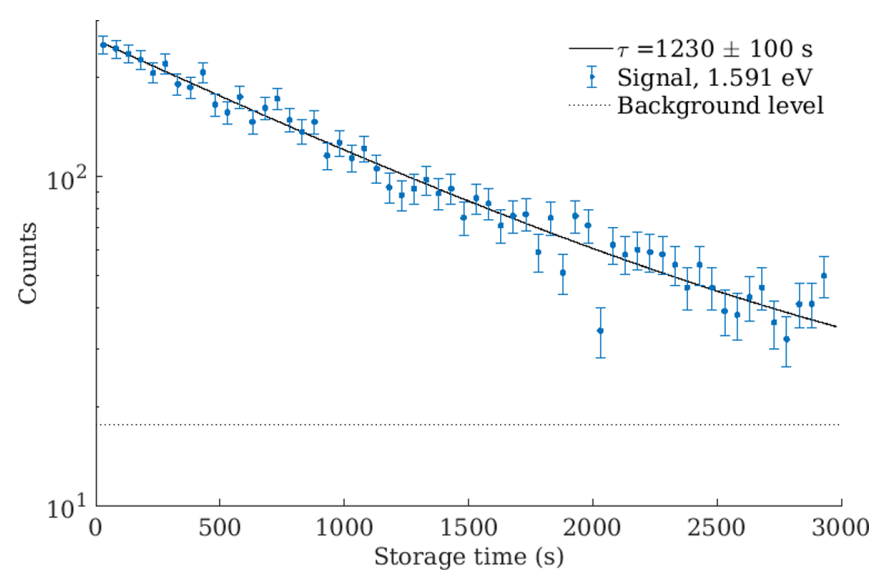

FIG. 4. Photodetachment signal from all states of $\mathrm{Ir}^{-}$using a photon energy of $1.591 \mathrm{eV}$ (arrow 1 in Fig. 3). The signal is recorded for one ion injection, and the data are binned using 50-s bins. The background is measured between each laser pulse and is fixed when making an exponential fit to the data giving a storage time of $1230 \pm 100 \mathrm{~s}$. overall storage time of the beam. The total number of detected photodetached neutrals is $\sim 5 \times 10^{3}$ in one measurement cycle. The total number of ions in the ring is $\sim 5.3 \times 10^{5}$, which corresponds to a $1-n A$ ion beam current almost completely filling up the storage ring. This means that the number of photodetached ions is only a small fraction (about $2 \%$ ) of the total ion beam. The photodetachment signal is monitored as a function of time, and an exponential function is fitted to the signal. A single exponential decay is characteristic for a beam that decays at a constant rate [21]. As shown in Fig. 4, a beam lifetime of $1230 \pm 100 \mathrm{~s}$ is determined, and it is evident from the data that there is a significant photodetachment signal left after $3000 \mathrm{~s}$ of ion-beam storage.

In order to photodetach ions in excited states only, leaving the ions in their ground states untouched, three different photon energies are used as illustrated in Fig. 3. A photon energy of $1.459 \mathrm{eV}$, corresponding to arrow no. 2, will photodetach all excited states. In order to measure the lifetime of state $A_{2}$, we store the ions for $2000 \mathrm{~s}$ and compare the time-dependent photodetachment signals with photon energies that correspond to arrow no. 1 vs arrow no. 2 in Fig. 3. We consider the signal after a few seconds of storage, and the only observed difference between the two measurements is that the count rate drops by a factor of $\sim 100$, and no difference in the lifetime of the signal is observed. This leads to the conclusion that the lifetime of excited-state $A_{2}$ is much longer than the measured beam storage lifetime of $1230 \pm 100 \mathrm{~s}$. When a photon energy lower than the binding energy of state $A_{2}$ is used, the long-lived decay disappears completely, thus, the assignment of this long lifetime to state $A_{2}$ is correct.

The next photon energy selected is $0.8856 \mathrm{eV}$, corresponding to arrow no. 3 in Fig. 3. This photon energy is well above the threshold energy needed to photodetach state $B_{3}$ as previously reported by Thøgersen et al. [23]. A clear photodetachment signal is observed using this photon energy. The collected signal is obtained over $12.5 \mathrm{~h}$ of data acquisition and shows a time dependence as illustrated in Fig. 5. An exponential function is fitted to the data, and a lifetime of 


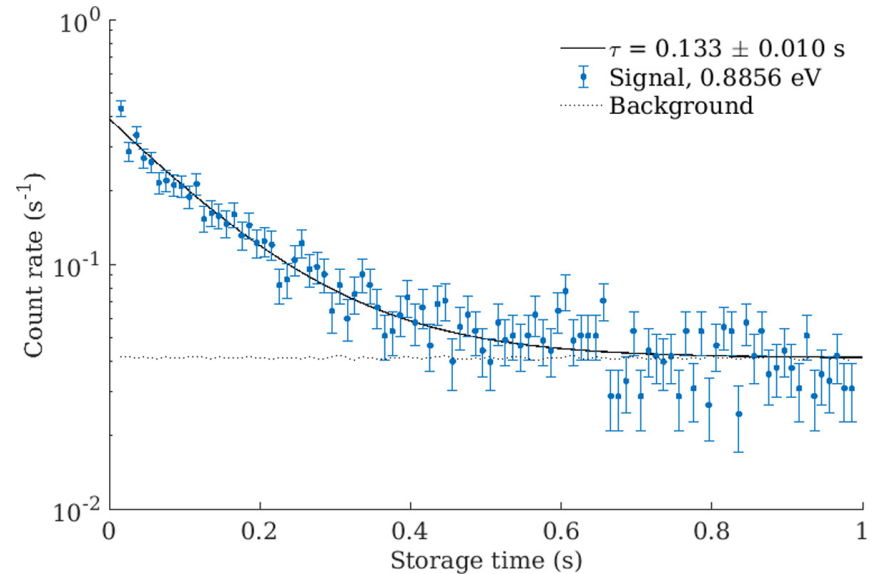

FIG. 5. Photodetachment signal using a photon energy of $0.8856 \mathrm{eV}$ (arrow 3 in Fig. 3). The signal is recorded during multiple injections in the storage ring. The background is measured between each laser pulse and fixed when making an exponential fit to the data. The signal is normalized to the gate size $(2 \mu \mathrm{s})$, the laser power, the number of measurement cycles, and the ion-beam current. An exponential fit gives a lifetime of $0.133 \pm 0.010 \mathrm{~s}$.

$133 \pm 10 \mathrm{~ms}$ is obtained. Complementary measurements are also performed with a photon energy of $0.6888 \mathrm{eV}$. This photon energy (not indicated in Fig. 3) is just above the threshold energy for photodetaching state $B_{3}$. The measurement yields a corresponding lifetime, but the signal-to-background ratio is low due to a slightly lower output from the OPO laser system. This measurement is, therefore, only used as a control, and the data are not used to determine the lifetime presented in this paper.

Careful investigations of the existence of a third excited bound state as predicted by our theoretical results are carried out using a photon energy of $0.6702 \mathrm{eV}$, corresponding to arrow no. 4 in Fig. 3. A small photodetachment signal is observed above the background level after $20 \mathrm{~h}$ of data collection which we attribute to a formerly unknown third excited state $C_{2}$ since this photon energy cannot photodetach ions in states $A_{2}$ or $B_{3}$. The time dependence of the signal is shown in Fig. 6. A single exponential function is fitted to the data yielding a lifetime of $172 \pm 35 \mathrm{~ms}$ for this third excited state with a binding energy $<0.6702 \mathrm{eV}$. The larger error compared to the measurement in Fig. 5 is due to the much weaker photodetachment signal. The signal-to-background ratio in Fig. 6 is about 1 , whereas it is around 10 in the data shown in Fig. 5. In order to compare the signal in Figs. 5 and 6, the data are normalized to obtain the count rate. The normalization factors include the signal gate size $(2 \mu \mathrm{s})$, the laser power, the number of measurement cycles, and the ion-beam current.

Returning to the decay presented in Fig. 5, the data shown in this figure are the sum of the decay from states $B_{3}$ and $C_{2}$. However, the signal from state $C_{2}$ is $\sim$ ten times smaller, and it was not possible to perform a fit to the sum of two exponential functions. Hence, we can state that the time-dependent signal shown in Fig. 5 using a photon energy of $0.8856 \mathrm{eV}$ is dominated by the decay of state $B_{3}$. Even a small contribution from the $C_{2}$ state of about $10 \%$ of the measured signal would not affect the measured lifetime within the given error bars.

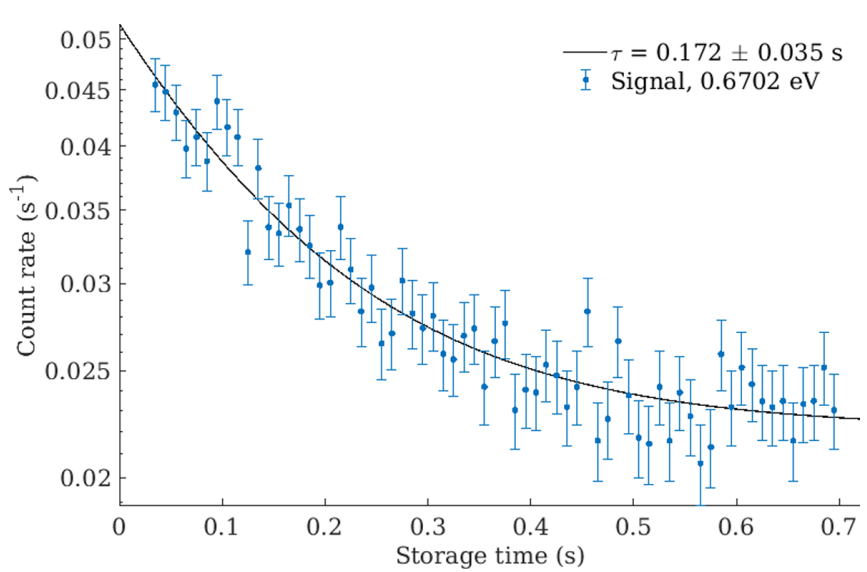

FIG. 6. Photodetachment signal using a photon energy of $0.6702 \mathrm{eV}$ (arrow 4 in Fig. 3). The signal is recorded during multiple injections in the storage ring. The signal is normalized to the gate size $(2 \mu \mathrm{s})$, the laser power, the number of measurement cycles, and the ion-beam current. An exponential fit is performed to the data resulting in a lifetime of $0.172 \pm 0.035 \mathrm{~s}$.

Finally, an investigation of the photon energy dependence of the photodetachment cross section in the range of 0.95 $1.1 \mathrm{eV}$ is performed as shown in Fig. 7. The signal is recorded for $8 \mathrm{~s}$, starting $22 \mathrm{~s}$ after the ions are injected into the storage ring, and the background is measured in between the laser pulses. The observed signal clearly reveals a $p$-wave photodetachment threshold and a fit to the Wigner law with $l=1$ (corresponding to the orbital angular momentum of the detached electron) results in a threshold value of $1.045 \pm$ $0.002 \mathrm{eV}$. We assign this threshold to the state labeled $A_{2}$ in Fig. 1. This result only differs by 1.5 standard deviations from that found by Lu et al. where the binding energy of this state is measured to be $1.04788 \pm 0.00002 \mathrm{eV}$ [27]. The two results can, therefore, be considered to be in fair agreement with each other. The background below the threshold is caused by collisional detachment and dark counts from the detector and is independent of the photon energy.

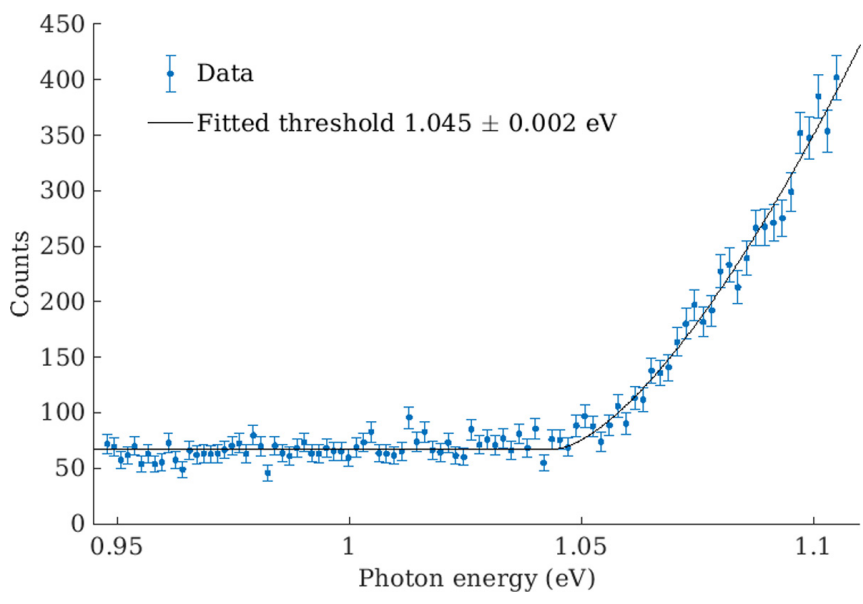

FIG. 7. Photodetachment yield as a function of photon energy. The signal is recorded during multiple injections in the storage ring. A fit to the Wigner law using $l=1$ is performed yielding a threshold value of $1.045 \pm 0.002 \mathrm{eV}$. The photodetachment signal is normalized to the laser power. 
TABLE I. Energy (in eV) spectrum of $\mathrm{Ir}^{-}$including the four bound states and the first two unbound states. Excitation energies are converted into binding energies by using the experimental electron affinity $-1.564057(11) \mathrm{eV}$ [27]. $L S$ composition of each level is also shown, highlighting the strong mixing among the $J=2$ levels.

\begin{tabular}{|c|c|c|c|c|c|}
\hline Label & $J$ & $\pi$ & $L S$ composition & $E_{b}(\mathrm{eV})$ & $E_{b}^{\text {expt }}(\mathrm{eV})$ \\
\hline \multicolumn{6}{|c|}{ VV correlation model } \\
\hline$X_{4}$ & 4 & + & ${ }^{3} F(87 \%)$ & & $\begin{array}{l}-1.56436(15)^{\mathrm{a}} \\
-1.564057(11)^{\mathrm{c}}\end{array}$ \\
\hline$A_{2}$ & 2 & + & ${ }^{1} D(43 \%)+{ }^{3} F(24 \%)+{ }^{3} P(19 \%)$ & -0.9742 & $\begin{array}{l}-1.045(2)^{\mathrm{b}} \\
-1.04788(2)^{\mathrm{c}}\end{array}$ \\
\hline$B_{3}$ & 3 & + & ${ }^{3} F(90 \%)$ & -0.7386 & $\begin{array}{l}-0.68635(5)^{\mathrm{d}} \\
-0.68535(4)^{\mathrm{c}}\end{array}$ \\
\hline$C_{2}$ & 2 & + & ${ }^{3} P(46 \%)+{ }^{3} F(43 \%)$ & -0.1434 & \\
\hline / & 0 & + & ${ }^{3} P(77 \%)+{ }^{1} S(12 \%)$ & 0.0884 & \\
\hline / & 1 & + & ${ }^{3} P(89 \%)$ & 0.2762 & \\
\hline \multicolumn{6}{|c|}{$\mathrm{VV}+\mathrm{CV}$ correlation model } \\
\hline$X_{4}$ & 4 & + & ${ }^{3} F(87 \%)$ & & $\begin{array}{l}-1.56436(15)^{\mathrm{a}} \\
-1.564057(11)^{\mathrm{c}}\end{array}$ \\
\hline$A_{2}$ & 2 & + & ${ }^{1} D(42 \%)+{ }^{3} F(24 \%)+{ }^{3} P(20 \%)$ & -0.9270 & $\begin{array}{l}-1.045(2)^{\mathrm{b}} \\
-1.04788(2)^{\mathrm{c}}\end{array}$ \\
\hline$B_{3}$ & 3 & + & ${ }^{3} F(90 \%)$ & -0.6939 & $\begin{array}{l}-0.68635(5)^{\mathrm{d}} \\
-0.68535(4)^{\mathrm{c}}\end{array}$ \\
\hline$C_{2}$ & 2 & + & ${ }^{3} P(47 \%)+{ }^{3} F(42 \%)$ & -0.0783 & \\
\hline l & 0 & + & ${ }^{3} P(79 \%)+{ }^{1} S(10 \%)$ & 0.1855 & \\
\hline / & 1 & + & ${ }^{3} P(89 \%)$ & 0.3478 & \\
\hline
\end{tabular}

${ }^{\text {a Reference [26]. }}$

${ }^{\mathrm{b}}$ This paper.

${ }^{\mathrm{c}}$ Reference [27].

${ }^{\mathrm{d}}$ Reference [23].

\section{THEORETICAL METHODS AND RESULTS}

The theoretical investigation carried out in this paper is performed with the variational and fully relativistic multiconfiguration MCDHF method [28], as implemented in Ref. [36] and $^{1}$ Ref. [29]. Following the self-consistent optimization of the wave functions, these codes also include methods for subsequent relativistic configuration-interaction (RCI) calculations. Such a final step is typically employed to further improve the accuracy of the wave functions by increasing the size of the atomic basis space, and the addition of the Breit interaction and leading-order quantum-electrodynamical (vacuum-polarization and electron-self-energy) corrections to the Hamiltonian.

The adopted atomic model targets the energy levels and radiative properties of all predicted to be bound states of $\mathrm{Ir}^{-}$. For the initial MCDHF, self-consistent optimization of the radial orbitals and the employed set of atomic basis functions were generated by considering the [Xe] $4 f^{14}\left\{5 d^{8} 6 s^{2}\right\}$ reference configuration. Here, the curly brackets define the valence $(V)$ electrons and the remaining [Xe] $4 f^{14}$ electrons are treated as a core $(C)$. Note that we use a nonrelativistic notation for simplicity with, e.g., $5 d$ implying both $5 d_{3 / 2}$ and $5 d_{5 / 2}$. The basis set is constructed by allowing for up to two simultaneous electron substitutions from the $5 s, 5 p, 5 d$, and $6 s$ orbitals with, at the most, one substitution from the $5 s$ or $5 p$ core orbitals. This basis space is constructed to

\footnotetext{
${ }^{1}$ Also available in Ref. [36].
}

account for $\mathrm{VV}$ and a dominant portion of $\mathrm{CV}$ correlation effects on the excitation energies and radiative transition properties. In a subsequent RCI calculation, the basis space was expanded by enlarging the single reference configuration to the [Xe] $4 f^{14}\left\{5 d^{8} 6 s^{2}, 5 d^{9} 6 s, 5 d^{10}\right\}$ multireference. Such an approach will effectively capture the most dominating higherorder correlation effects, and mainly important triple electron substitutions from the targeted states of the single reference. This correlation model, designed to treat electron interactions within and between the valence and the core shells, is referred to as VV + CV. Similar calculations are performed without including the electron substitutions from the $5 s, 5 p$ orbitals, thus, allowing only for substitutions from the $5 d, 6 s$ valence orbitals. This smaller correlation model, treating only interactions within the valence shells, is labeled VV.

The resulting energy levels are shown in Table I for the six lowest states of $\mathrm{Ir}^{-}$together with the $L S$ composition of each level. Excitation energies obtained with GRASP are converted into binding energies $\left(E_{b}\right)$ using the $-1.564057(11)-\mathrm{eV}$ experimental value of the Ir electron affinity [27]. The most important outcome of our calculations is the prediction of four bound states in $\mathrm{Ir}^{-}$as shown in Fig. 1. The figure shows that we can match the four bound states from the calculations with our experimentally observed states labeled $X_{4}, A_{2}, B_{3}$, and $C_{2}$. These states all belong to the [Xe] $4 f^{14} 5 d^{8} 6 s^{2}$ electronic ground configuration of $\mathrm{Ir}^{-}$.

We first discuss the $B_{3}$ state, which has previously been assigned with a ${ }^{3} F_{3}$ term. Its labeling is confirmed by our calculations which show that the ${ }^{3} F_{3}$ term accounts for $90 \%$ of the $L S$-term composition of the $B_{3}$ state wave function. 
TABLE II. Computed, MCDHF, and measured lifetimes of the three excited bound states of $\mathrm{Ir}^{-}$.

\begin{tabular}{lcc}
\hline \hline State & $\tau_{\mathrm{MCDHF}}$ & $\tau_{\text {expt }}$ \\
\hline$A_{2}$ & $>2500 \mathrm{~s}$ & $>1200 \mathrm{~s}$ \\
$B_{3}$ & $112(11) \mathrm{ms}$ & $133(10) \mathrm{ms}$ \\
$C_{2}$ & $161(8) \mathrm{ms}$ & $172(35) \mathrm{ms}$ \\
\hline \hline
\end{tabular}

The excitation energy of this almost pure ${ }^{3} F_{3}$ state then corresponds to the fine-structure energy splitting between the $J=3$ and the $J=4$ levels of the ${ }^{3} F$ term. This energy is close to the results from the two previous experimental studies [23,27], in particular, when taking into account $\mathrm{CV}$ correlation where the resulting binding energy is only 8 and $9 \mathrm{meV}$ away from the two experimental values. Here, the more accurate value using the CV correlation is expected for fine-structure splittings [37]. The discrepancy of $1 \mathrm{meV}$ of the two experimental results is too small for our calculations to resolve.

For states $A_{2}$ and $C_{2}$ both having $J=2$, the situation is more complex. These two states show a strong mixture of $L S$ terms, including ${ }^{3} P,{ }^{3} F$ in addition to ${ }^{1} D$ in the case of state $A_{2}$, which differ from the term designation of the ground state. This makes it more difficult to balance the effect of correlation and to provide accurate excitation energy since the fine structure of the singe ${ }^{3} F$ term is strongly perturbed by the ${ }^{1} D$ and is in a LS-coupling framework actually an interterm effect. The $A_{2}$ state was previously assigned to a ${ }^{3} P$ term. However, the present calculations indicate that only $20 \%$ of the wave function belongs to this term and that the dominating term is instead ${ }^{1} D$. The calculated and experimental excitation energies of this state differ by about $6 \%$ and $11 \%$ for the VV and $\mathrm{VV}+\mathrm{CV}$ correlation models, which suggest either that the treatment of the $\mathrm{CV}$ correlation between states $X_{4}$ and $A_{2}$ is unbalanced that the $\mathrm{CV}$ correlation is unconverged or both. The $C_{2}$ state, which is the second $J=2$ level, was previously predicted to be unbound, whereas the current paper indicates that it is, in fact, at least, loosely bound. This was predicted by our theoretical calculation and inspired our extended experimental search for the level.

Turning to radiative properties, the decay of the $B_{3}$ and $C_{2}$ states is dominated by $M 1$ transitions whereas state $A_{2}$ is very long lived and with a lowest-order allowed decay mode of $E 2$. The corresponding lifetimes are computed according to

$$
\tau(j)=\frac{1}{\sum_{i} A(j \rightarrow i)},
$$

where $A(j \rightarrow i)$ is the rate for the transition between the upper and the lower levels $j$ and $i$, respectively. The theoretical lifetimes are compared in Table II to the experimental values. For the $A_{2} \rightarrow X_{4}$ transition, the high sensitivity of the small $E 2$ decay rate to the correlation model and the gauge choice prevents us from providing a definite $\tau_{\mathrm{MCDHF}}^{A}$ value. Instead, the calculations yield a lower bound. To evaluate the lifetime of the $B_{3}$ state, the transition rates are rescaled using the experimental available binding energy (see, e.g., Ref. [32]). The lifetime of the $C_{2}$ state for which no experimental binding energy is available is computed by taking the average of the values resulting from the two correlation models. Uncertain- ties on $\tau_{\mathrm{MCDHF}}^{B}$ and $\tau_{\mathrm{MCDHF}}^{C}$ are estimated from the discrepancy between the two correlation models.

The overall agreement of the computed results with the experiment depends on the state and physical property of interest. The measurement confirms the theoretically predicted the $\tau^{B_{3}}<\tau^{C_{2}} \ll \tau^{A_{2}}$ lifetime relation. Furthermore, the theoretical investigations predict the existence of the third excited bound state (state $C_{2}$ ). This state is detected experimentally, and the measured lifetime agrees with the computed value.

Reaching high accuracy for the computed atomic properties of $\mathrm{Ir}^{-}$is particularly difficult, mainly because of the open $5 d$ shell of the ground configuration that induces a rapid growth of the number of basis states when further opening the $5 d$ and core orbitals to electron substitutions. This is likely the reason as to why, e.g., ab initio attempts on the group-VIII elements of the periodic table are so scarce [38,39]. Similar arguments apply to many of the neutron capture elements, in particular, lanthanides and actinides with open $f$-shell structures, which are of critical importance to the analysis of astrophysical events involving heavy elements, such as neutron-star mergers [40-43]. Difficulties, such as these motivate further developments of atomic structure computational strategies and codes to treat atomic systems with open $d$ and $f$ shells.

\section{CONCLUSIONS AND OUTLOOK}

We have performed photodetachment studies on $\mathrm{Ir}^{-}$using different photon energies and storage times at the DESIREE storage ring. MCDHF atomic structure calculations of the binding energies and lifetimes of the excited states were carried out in parallel to aid in the analysis and interpretation of the experimental results.

The binding energy of the first excited state $\left(A_{2}\right)$ is measured to be $1.045 \pm 0.002 \mathrm{eV}$, in good agreement with the recent measurement by Lu et al. [27]. Our calculations show that this state is a mix of three different $L S$-terms ${ }^{1} D(42 \%)+$ ${ }^{3} F(24 \%)+{ }^{3} P(20 \%)$, which contradicts its previous ${ }^{3} P$ term assignment [23]. The lifetime of this state is experimentally determined to be much longer than the experimental storage lifetime of $1230 \pm 100 \mathrm{~s}$, which is consistent with our theoretical estimates. A second excited state $\left(B_{3}\right)$, dominated by the ${ }^{3} F_{3}$ term in the theoretical results, is measured to have a lifetime of $133 \pm 10 \mathrm{~ms}$, in good agreement with the theoretically predicted value of $112 \pm 11 \mathrm{~ms}$. A previously unknown third excited state $\left(C_{2}\right)$ is theoretically predicted to be bound and later experimentally observed, which confirms its existence. This shows the importance of the close interplay between theory and experiment in the present paper. It also illustrates how careful and independent theoretical predictions can guide novel complex experiments, in general. The lifetime is experimentally determined to $172 \pm 35 \mathrm{~ms}$, in accordance with the computed $161 \pm 8$-ms value.

\section{ACKNOWLEDGMENTS}

This work was performed at the Swedish National Research Infrastructure, DESIREE (Swedish Research Council Contract No. 2017-00621). J.G., H.Z., H.T.S., and D.H. thank 
the Swedish Research Council for individual Project Grants (with Contracts No. 2020-05467, No. 2020-03437, No. 201804092, and No. 2016-0365). J.G. would like to acknowledge financial support from Project Grant "The New Milky Way"
(Grant No. 2013.0052) from the Knut and Alice Wallenberg Foundation. N.D.G. was supported, in part, by US National Science Foundation (NSF) Grant (Grant No. PHY-1707743). S.S. is a FRIA Grantee of the F.R.S-FNRS.
[1] D. J. Pegg, Structure and dynamics of negative ions, Rep. Prog. Phys. 67, 857 (2004).

[2] H. S. W. Massey, Negative Ions (Cambridge University Press, Cambridge, UK, 1950).

[3] C. W. Walter, N. D. Gibson, D. J. Matyas, C. Crocker, K. A. Dungan, B. R. Matola, and J. Rohlén, Candidate for Laser Cooling of a Negative Ion: Observations of Bound-Bound Transitions in $\mathrm{La}^{-}$, Phys. Rev. Lett. 113, 063001 (2014).

[4] R. C. Bilodeau and H. K. Haugen, Experimental Studies of $\mathrm{Os}^{-}$: Observation of a Bound-Bound Electric Dipole Transition in an Atomic Negative Ion, Phys. Rev. Lett. 85, 534 (2000).

[5] C. W. Walter, N. D. Gibson, Y.-G. Li, D. J. Matyas, R. M. Alton, S. E. Lou, R. L. Field, D. Hanstorp, L. Pan, and D. R. Beck, Experimental and theoretical study of bound and quasibound states of $\mathrm{Ce}^{-}$, Phys. Rev. A 84, 032514 (2011).

[6] A. Kellerbauer, A. Fischer, and U. Warring, Measurement of the Zeeman effect in an atomic anion: Prospects for laser cooling of Os $^{-}$, Phys. Rev. A 89, 043430 (2014).

[7] R. Tang, R. Si, Z. Fei, X. Fu, Y. Lu, T. Brage, H. Liu, C. Chen, and C. Ning, Candidate for Laser Cooling of a Negative Ion: High-Resolution Photoelectron Imaging of $\mathrm{Th}^{-}$, Phys. Rev. Lett. 123, 203002 (2019).

[8] E. Bäckström, D. Hanstorp, O. M. Hole, M. Kaminska, R. F. Nascimento, M. Blom, M. Björkhage, A. Källberg, P. Löfgren, P. Reinhed, S. Rosén, A. Simonsson, R. D. Thomas, S. Mannervik, H. T. Schmidt, and H. Cederquist, Storing keV Negative Ions for an Hour: The Lifetime of the Metastable ${ }^{2} P_{1 / 2}^{o}$ Level in ${ }^{32} \mathrm{~S}^{-}$, Phys. Rev. Lett. 114, 143003 (2015).

[9] M. Kamińska, V. T. Davis, O. M. Hole, R. F. Nascimento, K. C. Chartkunchand, M. Blom, M. Björkhage, A. Källberg, P. Löfgren, P. Reinhed, S. Rosén, A. Simonsson, R. D. Thomas, S. Mannervik, P. A. Neill, J. S. Thompson, H. T. Schmidt, H. Cederquist, and D. Hanstorp, Lifetime of the bound excited level in $\mathrm{Ni}^{-}$, Phys. Rev. A 93, 012512 (2016).

[10] K. C. Chartkunchand, M. Kamińska, E. K. Anderson, M. K. Kristiansson, G. Eklund, O. M. Hole, R. F. Nascimento, M. Blom, M. Björkhage, A. Källberg, P. Löfgren, P. Reinhed, S. Rosén, A. Simonsson, R. D. Thomas, S. Mannervik, V. T. Davis, P. A. Neill, J. S. Thompson, D. Hanstorp et al., Radiative lifetimes of the bound excited states of $\mathrm{Pt}^{-}$, Phys. Rev. A 94, 032501 (2016).

[11] L. M. Blau, R. Novick, and D. Weinflash, Lifetimes and Fine Structure of the Metastable Autoionizing $(1 s 2 s 2 p)^{4} P_{J}$ States of the Negative Helium Ion, Phys. Rev. Lett. 24, 1268 (1970).

[12] S. Mannervik, G. Astner, and M. Kisielinski, An optical transition in the negative lithium ion, J. Phys. B: At. Mol. Phys. 13, L441 (1980).

[13] F. Fleischer, K. Degreif, G. Gwinner, M. Lestinsky, V. Liechtenstein, F. Plenge, and D. Schwalm, Measurement of the Decay Rate of the Negative Ion of Positronium $\left(\mathrm{Ps}^{-}\right)$, Phys. Rev. Lett. 96, 063401 (2006).
[14] P. Balling, L. H. Andersen, T. Andersen, H. K. Haugen, P. Hvelplund, and K. Taulbjerg, Metastable Ion Lifetime Studies Utilizing a Heavy-Ion Storage Ring: Measurements on $\mathrm{Be}^{-}$, Phys. Rev. Lett. 69, 1042 (1992).

[15] T. Andersen, Lifetimes of negative ions determined in a storage ring, Phys. Scr. 1995, 230 (1995).

[16] S. P. Møller, Elisa, and electrostatic storage ring for atomic physics, Nucl. Instrum. Methods Phys. Res. Sec. A 394, 281 (1997).

[17] M. Dahan, R. Fishman, O. Heber, M. Rappaport, N. Altstein, D. Zajfman, and W. J. van der Zande, A new type of electrostatic ion trap for storage of fast ion beams, Rev. Sci. Instrum. 69, 76 (1998).

[18] P. Reinhed, A. Orbán, J. Werner, S. Rosén, R. D. Thomas, I. Kashperka, H. A. B. Johansson, D. Misra, L. Brännholm, M. Björkhage, H. Cederquist, and H. T. Schmidt, Precision Lifetime Measurements of $\mathrm{He}^{-}$in a Cryogenic Electrostatic Ion-Beam Trap, Phys. Rev. Lett. 103, 213002 (2009).

[19] Y. Nakano, Y. Enomoto, T. Masunaga, S. Menk, P. Bertier, and T. Azuma, Design and commissioning of the RIKEN cryogenic electrostatic ring (RICE), Rev. Sci. Instrum. 88, 033110 (2017).

[20] R. von Hahn, A. Becker, F. Berg, K. Blaum, C. Breitenfeldt, H. Fadil, F. Fellenberger, M. Froese, S. George, J. Göck, M. Grieser, F. Grussie, E. A. Guerin, O. Heber, P. Herwig, J. Karthein, C. Krantz, H. Kreckel, M. Lange, F. Laux et al., The cryogenic storage ring CSR, Rev. Sci. Instrum. 87, 063115 (2016).

[21] H. T. Schmidt, R. D. Thomas, M. Gatchell, S. Rosén, P. Reinhed, P. Löfgren, L. Brännholm, M. Blom, M. Björkhage, E. Bäckström, J. D. Alexander, S. Leontein, D. Hanstorp, H. Zettergren, L. Liljeby, A. Källberg, A. Simonsson, F. Hellberg, S. Mannervik, M. Larsson et al., First storage of ion beams in the double electrostatic ion-ring experiment: DESIREE, Rev. Sci. Instrum. 84, 055115 (2013).

[22] E. P. Wigner, On the behavior of cross sections near thresholds, Phys. Rev. 73, 1002 (1948).

[23] J. Thøgersen, M. Scheer, L. D. Steele, H. K. Haugen, and W. P. Wijesundera, Two-Photon Detachment of Negative Ions Via Magnetic Dipole Transitions, Phys. Rev. Lett. 76, 2870 (1996).

[24] C. S. Feigerle, R. R. Corderman, S. V. Bobashev, and W. C. Lineberger, Binding energies and structure of transition metal negative ions, J. Chem. Phys. 74, 1580 (1981).

[25] B. J. Davies, C. W. Ingram, D. J. Larson, and U. Ljungblad, The electron affinity of iridium, J. Chem. Phys. 106, 5783 (1997).

[26] R. C. Bilodeau, M. Scheer, H. K. Haugen, and R. L. Brooks, Near-threshold laser spectroscopy of iridium and platinum negative ions: Electron affinities and the threshold law, Phys. Rev. A 61, 012505 (1999). 
[27] Y. Lu, J. Zhao, R. Tang, X. Fu, and C. Ning, Measurement of electron affinity of iridium atom and photoelectron angular distributions of iridium anion, J. Chem. Phys. 152, 034302 (2020).

[28] C. F. Fischer, M. Godefroid, T. Brage, P. Jönsson, and G. Gaigalas, Advanced multiconfiguration methods for complex atoms: I. Energies and wave functions, J. Phys. B: At. Mol. Opt. Phys. 49, 182004 (2016).

[29] C. F. Fischer, G. Gaigalas, P. Jönsson, and J. Bieroń, Grasp2018-a fortran 95 version of the general relativistic atomic structure package, Comput. Phys. Commun. 237, 184 (2019).

[30] C. F. Fischer, Correlation in negative ions, Phys. Scr. 40, 25 (1989).

[31] R. Si, C. Y. Zhang, K. Yao, T. Brage, C. Y. Chen, and Y. M. Zou, Persistent discrepancy between experimental and theoretical lifetimes for $\mathrm{Ni}^{-}$, Phys. Rev. A 95, 042504 (2017).

[32] Y. Su, R. Si, K. Yao, and T. Brage, The structure and radiative lifetimes of negative ions homologous to N-, J. Phys. B: At. Mol. Opt. Phys. 52, 125002 (2019).

[33] T. Carette, C. Drag, O. Scharf, C. Blondel, C. Delsart, C. Froese Fischer, and M. Godefroid, Isotope shift in the sulfur electron affinity: Observation and theory, Phys. Rev. A 81, 042522 (2010).

[34] R. D. Thomas, H. T. Schmidt, G. Andler, M. Björkhage, M. Blom, L. Brännholm, E. Bäckström, H. Danared, S. Das, N. Haag, P. Halldén, F. Hellberg, A. I. S. Holm, H. A. B.
Johansson, A. Källberg, G. Källersjö, M. Larsson, S. Leontein, L. Liljeby, P. Löfgren et al., The double electrostatic ion ring experiment: A unique cryogenic electrostatic storage ring for merged ion-beams studies, Rev. Sci. Instrum. 82, 065112 (2011).

[35] National electrostatic corporation, USA. SNICS—-source of negative ions by cesium sputtering.

[36] The GRASP2018 program suite: www.github.com/compas/grasp

[37] C. Froese-Fischer and T. Brage, Computational Atomic Structure: An MCHF Approach (CRC Press, Boca Raton, FL, 1997).

[38] S. Schiffmann and M. Godefroid, Electronic isotope shift factors for the Ir $5 d^{7} 6 s^{24} f_{9 / 2} \rightarrow$ (odd, $j=9 / 2$ ) line at $247.587 \mathrm{~nm}$, J. Quant. Spectrosc. Radiat. Transfer 258, 107332 (2021).

[39] P. Palmeri, P. Quinet, and S. Bouazza, MCDHF calculations of isotope shifts of even-parity fine-structure levels in neutral osmium, J. Quant. Spectrosc. Radiat. Transfer 185, 70 (2016).

[40] M. Tanaka, Kilonova/Macronova emission from compact binary mergers, Adv. Astron. 2016, 1 (2016).

[41] F.-K. Thielemann, M. Eichler, I. V. Panov, and B. Wehmeyer, Neutron star mergers and nucleosynthesis of heavy elements, Annu. Rev. Nucl. Part. Sci. 67, 253 (2017).

[42] B. D. Metzger, Kilonovae, Liv. Rev. Relativ. 23, 1 (2020).

[43] J. J. Cowan, C. Sneden, J. E. Lawler, A. Aprahamian, M. Wiescher, K. Langanke, G. Martínez-Pinedo, and F.K. Thielemann, Origin of the heaviest elements: The rapid neutron-capture process, Rev. Mod. Phys. 93, 015002 (2021). 